\title{
Optimal Control for Degenerate Parabolic Equations \\ with Logistic Growth*
}

\author{
by \\ Suzanne M. Lenhart ${ }^{1}$ and Jiongmin Yong ${ }^{2}$
}

\begin{abstract}
This paper considers the optimal control of a degenerate parabolic partial differential equation governing a diffusive population with logistic growth terms. Assuming this population causes damage to forest and agricultural land, the optimal control is the trapping rate and the cost functional is a combination of the damage and trapping costs. We prove existence, uniqueness, and regularity results for this degenerate parabolic equation. The vanishing viscosity method is used to obtain the existence result. The optimal control is characterized in terms of the solution of the optimality system, which is the state equation coupled with the adjoint equation. Uniqueness for the solutions of the optimality system is valid for a sufficiently small time interval due to the opposite time orientations of the two equations involved.
\end{abstract}

Keywords. degenerate parabolic equations, logistic growth, optimal control, trapping strategy.

AMS Mathematics subject classification. 35K65, 49J20, 49K20, 92D25.

* This work was completed while the authors were visiting the Institute for Mathematics and Applications (IMA), University of Minnesota, USA and partially supported by the IMA. The first author was also partially supported by a grant from NSF and the second author was also partially supported by the NSF of China under Grant 19131050 and the Fok Ying Tung Education Foundation.

1 Department of Mathematics, University of Tennessee, Knoxville, TN 37996

2 Department of Mathematics, Fudan University, Shanghai 200433, China. 


\section{$\S 1$. Introduction.}

This paper concerns the application of a distributed parameter control model for the management of diffusive populations, whose growth is governed by logistic terms. The migration of small mammal species such as beavers, raccoons, and muskrats have been modeled by parabolic diffusion equations; see the classic work by Skellam on diffusing populations [16]. It has also been shown that the growth of such mammals follow logistic growth patterns [8]. Due to environmental heterogeneity and barriers, the diffusion may be degenerate in some locations. These small mammals are often found in conflict with human interests due to the damage they inflict on timber and agricultural land $[6,13]$. Our model uses a degenerate parabolic partial differential equation (PDE) with logistic growth terms to characterize the optimal strategy in trapping a proportion of such a nuisance population in order to minimize damage and trapping costs.

The population density is governed by the following state equation:

$$
z_{t}-\sum_{i, j=1}^{n}\left(a_{i j}(x) z_{x_{i}}\right)_{x_{j}}=z(a-b z-p), \quad \text { in } \Omega_{T} \equiv \Omega \times(0, T),
$$

with boundary conditions $z=0$ or no flux boundary conditions. The problem is posed in $\Omega \times(0, T)$ with $\Omega \subset \mathbb{R}^{n}$. For practical applications, the habitat sits in $\mathbb{R}^{2}$, i.e., $\Omega \subset \mathbb{R}^{2}$. The diffusion coefficients $\left(a_{i j}(x)\right)$ may be degenerate at some locations. We assume the initial distribution is known. The proportion $p(x, t)$ of the population to be trapped is the control in our problem. We can treat zero Dirichlet $(z=0)$ or Neumann (no flux) boundary conditions. If the exterior of the domain is completely hostile to the population and the species go out there anyway, then zero Dirichlet boundary conditions are reasonable [14]. Otherwise if the population does not go out to the boundary, then "no flux" Neumann boundary conditions are valid.

Choosing controls in an appropriate class, we seek to minimize the cost functional:

$$
J(p)=\int_{\Omega_{T}} e^{-r t}\left(\frac{1}{2} \alpha z^{2}+\beta p^{2} z\right) d x d t
$$

The the $z^{2}$ term represents the density dependent damage due the nuisance species. The amount of the population trapped is $p z$ and the unit trapping cost at level $p$ is $\beta p$; these 
two factors multiply to give the cost of trapping in the cost functional. The $e^{-r t}$ term is included for discounting the value of the future cost accruing over the given finite planning horizon. We will characterize the optimal control that minimizes the cost functional.

Section 2 gives the precise formulation of the problem in terms of weak solutions of the state equation. In section 3, we prove existence, uniqueness, and regularity results for weak solutions of the state equation. The vanishing viscosity method is used to obtain the existence result. In section 4, the existence of optimal controls is obtained under the assumption that the set where the diffusion vanishes has measure zero. Also, the optimal control is characterized in terms of the unique solution of the optimality system, which consists of the state equation coupled with an adjoint equation. The optimality system is derived by differentiating the cost functional with respect to control at an optimal control. Sections 2 through 4 consider the Dirichlet boundary condition case, while section 5 treats the Neumann boundary condition case.

Background concerning biology and economics behind diffusion population models with logistic growth terms can be found in the books by Murray [14] and Clark [2]. For background on control and PDEs, see the books by Fife [4] and Lions [12]. Leung and Stojanovic [10] have optimal control results for uniformly elliptic PDE with logistic growth terms, with a different cost functional than that used here. He, Leung, and Stojanovic

[5] considered optimal harvesting control of a periodic parabolic system for a competitive model with maximization of profit. Bhat and Lenhart [9] treated this wildlife management problem in the case of constant diffusion. Their work easily generalizes to the nondegenerate diffusion case, but not to the degenerate case. They demonstrated their model with a numerical simulation using beaver population data from the New York State Department of Environmental Conservation. The simulation is unusual due the opposite time orientations of the state and the adjoint equations.

\section{$\S 2$. Formulation of the Problem.}

In this section, we will formulate our optimal control problem. In what follows, we let $\Omega \subset \mathbb{R}^{n}$ be an open set with a smooth boundary $\partial \Omega$ and $T>0$ be a fixed constant. For 
any $t \in[0, T]$, denote $\Omega_{t}=\Omega \times(0, t)$. Our state equation is the following:

$$
\left\{\begin{array}{l}
z_{t}-\sum_{i, j=1}^{n}\left(a_{i j}(x) z_{x_{i}}\right)_{x_{j}}=z(a-b z-p), \quad \text { in } \Omega_{T} \\
\left.z\right|_{\partial \Omega}=0 \\
\left.z\right|_{t=0}=z_{0}(x)
\end{array}\right.
$$

where $z$ is the state, $z_{0}$ and $p$ are the initial state and the control, respectively. We make the following assumptions:

(A1) Functions $a_{i j}: \bar{\Omega} \rightarrow \mathbb{R}$ are $C^{1}$ and the matrix $\left\{a_{i j}(x)\right\}$ is symmetric positive semidefinite for all $x \in \bar{\Omega}$. Moreover,

$$
\operatorname{meas}\left\{x \in \Omega \mid \operatorname{det}\left\{a_{i j}(x)\right\}=0\right\}=0,
$$

and there exists a $C^{1}$ function $\sigma: \bar{\Omega} \rightarrow \mathbb{R}^{n \times n}$, such that

$$
\left\{a_{i j}(x)\right\}=\sigma^{*}(x) \sigma(x), \quad x \in \bar{\Omega} .
$$

(A2) $a$ and $b$ appearing in (2.1) are nonnegative constants.

We point out that one may let $a$ and $b$ be $L^{\infty}\left(\Omega_{T}\right)$ functions. Letting them be constants is just for simplifying the notation. Also, we may let $a_{i j}$ be time dependent.

Next, let us define $H_{\sigma}(\Omega)$ to be the completion of $C_{0}^{\infty}(\Omega)$ under norm

$$
\|z\|_{H_{\sigma}(\Omega)}=\left\{\|z\|_{L^{2}(\Omega)}^{2}+\|\sigma \nabla z\|_{L^{2}(\Omega)}^{2}\right\}^{1 / 2}
$$

Clearly, $H_{\sigma}(\Omega)$ is a Hilbert space. See $[15$, p.28] for similar solution spaces. We denote its dual by $H_{\sigma}(\Omega)^{*}$. Then, we see that the embeddings $H_{\sigma}(\Omega) \hookrightarrow L^{2}(\Omega) \hookrightarrow H_{\sigma}(\Omega)^{*}$ are continuous. Now, we introduce the following sets:

$$
\begin{aligned}
& \mathcal{Z}_{K}=\left\{z \in C(\bar{\Omega})|0<z(x) \leq K, \quad x \in \Omega, \quad z|_{\partial \Omega}=0\right\}, \quad \mathcal{Z}=\bigcup_{K>0} \mathcal{Z}_{K} \\
& \mathcal{P}_{M}=\left\{p \in L^{\infty}\left(\Omega_{T}\right) \mid 0 \leq p(x, t) \leq M, \quad \text { a.e. }(x, t) \in \Omega_{T}\right\}, \quad \mathcal{P}=\bigcup_{M>0} \mathcal{P}_{M} .
\end{aligned}
$$

The control $p$ is taken from the set $\mathcal{P}$ and the initial state $z_{0}$ is taken from $\mathcal{Z}$. In $\S 3$, we will show that under assumptions (A1)-(A2), for any $\left(z_{0}, p\right) \in \mathcal{Z} \times \mathcal{P}$, there exists a unique 
solution $z$ in some weak sense, which turns out to be in $L^{\infty}\left(\Omega_{T}\right)$. We refer to this $z$ as the state corresponding to the given initial state $z_{0}$ and the control $p$. Thus, we may define the following cost functional:

$$
J(p)=\int_{\Omega_{T}} e^{-r t}\left(\frac{1}{2} \alpha z^{2}+\beta p^{2} z\right) d x d t
$$

Here we make the following assumption:

(A3) $\alpha, \beta$ and $r$ are constants with constraints:

$$
\alpha, r \geq 0, \quad \beta \geq \beta_{0}>0 .
$$

Again, we may let $\alpha, \beta$ and $r$ be $L^{\infty}\left(\Omega_{T}\right)$ functions satisfying (2.7) almost everywhere on $\Omega_{T}$. Clearly, under assumptions (A1)-(A3), for any given $z_{0} \in \mathcal{Z}$ and $p \in \mathcal{P}$, the cost functional $J(p)$ is well-defined. Our optimal control problem can be stated as follows:

Problem $P_{K, M}$. Let $K, M>0$ and $z_{0} \in \mathcal{Z}_{K}$ be given. Find an element $\bar{p} \in \mathcal{P}_{M}$, such that

$$
J(\bar{p})=\inf _{p \in \mathcal{P}_{M}} J(p)
$$

Any element $\bar{p} \in \mathcal{P}$ satisfying (2.8) is called an optimal control and the corresponding state, denoted by $\bar{z}$, is called an optimal state.

\section{$\S 3$. State Equation.}

In this section, we prove the existence and uniqueness of solutions to the state equation. To start with, let us introduce the following definition which is adapted from [7].

Definition 3.1. Let $\left(z_{0}, p\right) \in \mathcal{Z} \times \mathcal{P}$ be given. A function $z \in L^{2}\left(0, T ; H_{\sigma}(\Omega)\right)$ is called a solution of (2.1) if the following holds:

$$
\begin{gathered}
\int_{\Omega_{T}}\left[-z \eta_{t}+\sum_{i, j=1}^{n} a_{i j} z_{x_{i}} \eta_{x_{j}}\right] d x d t=\int_{\Omega} z_{0}(x) \eta(x, 0) d x+\int_{\Omega_{T}}(a-b z-p) z \eta d x d t \\
\forall \eta \in W_{2}^{0,1}\left(\Omega_{T}\right) \bigcap L^{2}\left(0, T ; H_{\sigma}(\Omega)\right), \quad \text { with }\left.\eta\right|_{t=T}=0,\left.\quad \eta\right|_{\partial \Omega}=0 .
\end{gathered}
$$

Our main result of this section is the following. 
Theorem 3.2. Let assumptions (A1)-(A2) hold. Then, we have the following conclusions:

(i) For any $z_{0} \in \mathcal{Z}$ and $p \in \mathcal{P}$, there exists a unique solution $z \in L^{2}\left([0, T] ; H_{\sigma}(\Omega)\right) \bigcap$ $L^{\infty}\left(\Omega_{T}\right) \bigcap C\left([0, T] ; L^{2}(\Omega)\right)$, with $z_{t} \in L^{2}\left(0, T ; H_{\sigma}(\Omega)^{*}\right)$, of the state equation (2.1) satisfying the following:

$$
\frac{1}{2} z_{0}(x) e^{-\mu t} \leq z(x, t) \leq \frac{a}{b} \vee\left\|z_{0}\right\|_{L^{\infty}(\Omega)}, \quad \text { a.e. }(x, t) \in \Omega_{T},
$$

with $\mu=b\left(\frac{a}{b} \vee\left\|z_{0}\right\|_{L^{\infty}(\Omega)}\right)+\|p\|_{L^{\infty}\left(\Omega_{T}\right)}-a$ and

$$
\begin{gathered}
\left\|z_{t}\right\|_{L^{2}\left(0, T ; H_{\sigma}(\Omega)^{*}\right)}+\|z\|_{C\left([0, T] ; L^{2}(\Omega)\right)}+\|\sigma \nabla z\|_{L^{2}\left(\Omega_{T}\right)} \\
\leq C\left(1+\|p\|_{L^{1}\left(\Omega_{T}\right)}\right)
\end{gathered}
$$

with $C=C\left(\left\|z_{0}\right\|_{L^{\infty}(\Omega)}\right)$.

(ii) Let $\left(z_{0}, p\right),\left(\widehat{z}_{0}, \widehat{p}\right) \in \mathcal{Z} \times \mathcal{P}$ and $z, \widehat{z}$ are corresponding solutions of (2.1). Then,

$$
\begin{gathered}
\left\|(z-\widehat{z})_{t}\right\|_{L^{2}\left(0, T ; H_{\sigma}(\Omega)^{*}\right)}+\|z-\widehat{z}\|_{C\left([0, T] ; L^{2}(\Omega)\right)}+\|\sigma \nabla(z-\widehat{z})\|_{L^{2}\left(\Omega_{T}\right)} \\
\leq C\left\{\left\|z_{0}-\widehat{z}_{0}\right\|_{L^{2}(\Omega)}+\|p-\widehat{p}\|_{L^{1}\left(\Omega_{T}\right)}\right\}
\end{gathered}
$$

with $C=C\left(\left\|z_{0}\right\|_{L^{\infty}(\Omega)},\left\|\widehat{z}_{0}\right\|_{L^{\infty}(\Omega)}\right)$.

(iii) Furthermore, if $\left(z_{0}, p\right) \in\left(\mathcal{Z} \cap H_{\sigma}(\Omega)\right) \times \mathcal{P}$, then the corresponding solution $z \in C\left([0, T] ; H_{\sigma}(\Omega)\right)$ with $z_{t} \in L^{2}\left(\Omega_{T}\right)$ and satisfies the following:

$$
\left\|z_{t}\right\|_{L^{2}\left(\Omega_{T}\right)}+\|\sigma \nabla z\|_{C\left([0, T] ; L^{2}(\Omega)\right)} \leq\left\|\sigma \nabla z_{0}\right\|_{L^{2}(\Omega)}+C,
$$

with $C=C\left(\left\|z_{0}\right\|_{L^{\infty}(\Omega)},\|p\|_{L^{2}\left(\Omega_{T}\right)}\right)$.

(iv) Finally, if $\left(z_{0}, p\right),\left(\widehat{z}_{0}, \widehat{p}\right) \in\left(\mathcal{Z} \cap H_{\sigma}(\Omega)\right) \times \mathcal{P}$, then the corresponding solutions $z$ and $\widehat{z}$ satisfy

$$
\begin{aligned}
\left\|z_{t}-\widehat{z}_{t}\right\|_{L^{2}\left(\Omega_{T}\right)}+ & \|\sigma \nabla(z-\widehat{z})\|_{C\left([0, T] ; L^{2}(\Omega)\right)} \\
& \leq C\left(\left\|\sigma \nabla\left(z_{0}-\widehat{z}_{0}\right)\right\|_{L^{2}(\Omega)}+\left\|z_{0}-\widehat{z}_{0}\right\|_{L^{2}(\Omega)}+\|p-\widehat{p}\|_{L^{2}(\Omega)}\right),
\end{aligned}
$$

with $C=C\left(\left\|z_{0}\right\|_{L^{\infty}(\Omega)},\left\|\widehat{z}_{0}\right\|_{L^{\infty}(\Omega)},\|p\|_{L^{\infty}\left(\Omega_{T}\right)} \wedge\|\widehat{p}\|_{L^{\infty}\left(\Omega_{T}\right)}\right)$.

Proof. First, we let $\left(z_{0}, p\right) \in\left(C_{0}^{\infty}(\Omega) \cap \mathcal{Z}\right) \times\left(C^{\infty}\left(\bar{\Omega}_{T}\right) \bigcap \mathcal{P}\right)$. For any $\varepsilon>0$, we consider the following approximating state equation:

$$
\left\{\begin{array}{l}
z_{t}^{\varepsilon}-\varepsilon \Delta z^{\varepsilon}-\sum_{i, j=1}^{n}\left(a_{i j}(x) z_{x_{i}}^{\varepsilon}\right)_{x_{j}}=z^{\varepsilon}\left(a-b z^{\varepsilon}-p\right), \quad \text { in } \Omega_{T} \\
\left.z^{\varepsilon}\right|_{\partial \Omega}=0 \\
\left.z^{\varepsilon}\right|_{t=0}=z_{0}(x) .
\end{array}\right.
$$


This problem admits a unique classical solution $z^{\varepsilon}$. Similar to [9], we can prove that

$$
0 \leq z^{\varepsilon}(x, t) \leq \frac{a}{b} \vee\left\|z_{0}\right\|_{L^{\infty}(\Omega)}
$$

Next, since $z_{0}>0$ in the interior of $\Omega$ and vanishes on the boundary $\partial \Omega$, we can find a solution $y^{\varepsilon} \in W_{0}^{2, p}(\Omega)$ to the following problem ([1]):

$$
\left\{\begin{array}{l}
-\varepsilon \Delta y^{\varepsilon}-\sum_{i, j=1}^{n}\left(a_{i j} y_{x_{i}}^{\varepsilon}\right)_{x_{j}} \leq 0, \quad \text { in } \Omega \\
\frac{1}{2} z_{0} \leq y^{\varepsilon} \leq z_{0}, \quad \text { in } \Omega \\
\left.y^{\varepsilon}\right|_{\partial \Omega}=0
\end{array}\right.
$$

We let $\mu=b\left(\frac{a}{b} \vee\left\|z_{0}\right\|_{L^{\infty}(\Omega)}\right)+\|p\|_{L^{\infty}(\Omega)}-a$ and let

$$
\widetilde{z}^{\varepsilon}(x, t)=e^{-\mu t} y^{\varepsilon}(x), \quad(x, t) \in \Omega_{T}
$$

By (3.8), we have

$$
\left\{\begin{array}{l}
\left(\widetilde{z}^{\varepsilon}-z^{\varepsilon}\right)_{t}-\varepsilon \Delta\left(\widetilde{z}^{\varepsilon}-z^{\varepsilon}\right)-\sum_{i, j=1}^{n}\left[a_{i j}\left(\widetilde{z}^{\varepsilon}-z^{\varepsilon}\right)_{x_{i}}\right]_{x_{j}} \leq-\mu\left(\widetilde{z}^{\varepsilon}-z^{\varepsilon}\right), \quad \text { in } \Omega_{T} \\
\left.\left(\widetilde{z}^{\varepsilon}-z^{\varepsilon}\right)\right|_{\partial \Omega}=0 \\
\left.\left(\widetilde{z}^{\varepsilon}-z^{\varepsilon}\right)\right|_{t=0} \leq 0 .
\end{array}\right.
$$

By maximum principle, we have

$$
z^{\varepsilon}(x, t) \geq \widetilde{z}^{\varepsilon}(x, t)=e^{-\mu t} y^{\varepsilon}(x) \geq \frac{1}{2} e^{-\mu t} z_{0}(x), \quad(x, t) \in \Omega_{T}
$$

Now, we let $\left(\widehat{z}_{0}, \widehat{p}\right)$ be another pair of smooth data, and $\widehat{z}^{\varepsilon}$ be the corresponding solution of (3.7). Then, the difference $w=z^{\varepsilon}-\widehat{z}^{\varepsilon}$ satisfies

$$
\left\{\begin{array}{l}
w_{t}-\varepsilon \Delta w-\sum_{i, j=1}^{n}\left(a_{i j}(x) w_{x_{i}}\right)_{x_{j}} \\
=\left[a-b\left(z^{\varepsilon}+\widehat{z}^{\varepsilon}\right)-p\right] w-(p-\widehat{p}) \widehat{z}^{\varepsilon}, \quad \text { in } \Omega_{T} \\
\left.w\right|_{\partial \Omega}=0, \\
\left.w\right|_{t=0}=z_{0}(x)-\widehat{z}_{0}(x) .
\end{array}\right.
$$


Then, multiply the equation by $w$ and integrate it on $\Omega$, we have

$$
\begin{gathered}
\frac{1}{2} \frac{d}{d t}\|w(\cdot, t)\|_{L^{2}(\Omega)}^{2}+\varepsilon\|\nabla w(\cdot, t)\|_{L^{2}(\Omega)}^{2}+\|\sigma \nabla w(\cdot, t)\|_{L^{2}(\Omega)}^{2} \\
\leq C\left(\|w(\cdot, t)\|_{L^{2}(\Omega)}^{2}+\|p(\cdot, t)-\widehat{p}(\cdot, t)\|_{L^{1}(\Omega)}\right) .
\end{gathered}
$$

By Gronwall's inequality, we have

$$
\begin{aligned}
& \left\|z^{\varepsilon}-\widehat{z}^{\varepsilon}\right\|_{C\left([0, T] ; L^{2}(\Omega)\right)}^{2}+\varepsilon\left\|\nabla\left(z^{\varepsilon}-\widehat{z}^{\varepsilon}\right)\right\|_{L^{2}\left(\Omega_{T}\right)}^{2}+\left\|\sigma \nabla\left(z^{\varepsilon}-\widehat{z}^{\varepsilon}\right)\right\|_{L^{2}\left(\Omega_{T}\right)}^{2} \\
& \quad \leq C\left\{\left\|z_{0}-\widehat{z}_{0}\right\|_{L^{2}(\Omega)}^{2}+\|p-\widehat{p}\|_{L^{1}\left(\Omega_{T}\right)}\right\},
\end{aligned}
$$

with $C=C\left(\left\|z_{0}\right\|_{L^{\infty}(\Omega)},\left\|\widehat{z}_{0}\right\|_{L^{\infty}(\Omega)}\right)$. Next, for any $\varphi \in C_{0}^{\infty}\left(\Omega_{T}\right)$, we have

$$
\int_{\Omega_{T}} w_{t} \varphi=\int_{\Omega_{T}}\left\{-\varepsilon \nabla w \cdot \nabla \varphi-\sigma \nabla w \cdot \sigma \nabla \varphi+\left[a-b\left(z^{\varepsilon}+\widehat{z}^{\varepsilon}\right)-p\right] w \varphi\right\}
$$

Then, by (3.15), it is clear that

$$
\left\|\left(z^{\varepsilon}-\widehat{z}^{\varepsilon}\right)_{t}\right\|_{L^{2}\left(0, T ; H_{\sigma}(\Omega)^{*}\right)} \leq C\left\{\left\|z_{0}-\widehat{z}_{0}\right\|_{L^{2}(\Omega)}^{2}+\|p-\widehat{p}\|_{L^{1}\left(\Omega_{T}\right)}\right\}
$$

with a constant similar to (3.15).

Now, multiply the equation in (3.13) by $w_{t}$ and integrate it over $\Omega$, we have

$$
\begin{aligned}
& \left\|w_{t}(\cdot, t)\right\|_{L^{2}(\Omega)}^{2}+\frac{\varepsilon}{2} \frac{d}{d t}\|\nabla w(\cdot, t)\|_{L^{2}(\Omega)}^{2}+\frac{1}{2} \frac{d}{d t}\|\sigma \nabla w\|_{L^{2}(\Omega)}^{2} \\
& \leq\left[a+b\left(\left\|z^{\varepsilon}\right\|_{L^{\infty}\left(\Omega_{T}\right)}+\left\|\widehat{z}^{\varepsilon}\right\|_{L^{\infty}\left(\Omega_{T}\right)}\right)+\|p\|_{L^{\infty}\left(\Omega_{T}\right)}\right]\|w(\cdot, t)\|_{L^{2}(\Omega)}\left\|w_{t}(\cdot, t)\right\|_{L^{2}(\Omega)} \\
& \quad+\left\|\widehat{z}^{\varepsilon}\right\|_{L^{\infty}\left(\Omega_{T}\right)}\|p(\cdot, t)-\widehat{p}(\cdot, t)\|_{L^{2}(\Omega)}\left\|w_{t}(\cdot, t)\right\|_{L^{2}(\Omega)} .
\end{aligned}
$$

Then, by Cauchy-Schwartz inequality and Gronwall's inequality, we obtain

$$
\begin{aligned}
& \left\|z_{t}^{\varepsilon}-\widehat{z}_{t}^{\varepsilon}\right\|_{L^{2}\left(\Omega_{T}\right)}^{2}+\varepsilon\left\|\nabla\left(z^{\varepsilon}-\widehat{z}^{\varepsilon}\right)\right\|_{C\left([0, T] ; L^{2}(\Omega)\right)}^{2}+\left\|\sigma \nabla\left(z^{\varepsilon}-\widehat{z}^{\varepsilon}\right)\right\|_{C\left([0, T] ; L^{2}(\Omega)\right)}^{2} \\
& \leq \varepsilon\left\|\nabla\left(z_{0}-\widehat{z}_{0}\right)\right\|_{L^{2}(\Omega)}^{2}+C\left(\left\|\sigma \nabla\left(z_{0}-\widehat{z}_{0}\right)\right\|_{L^{2}(\Omega)}^{2}\right. \\
& \left.\quad+\left\|z_{0}-\widehat{z}_{0}\right\|_{L^{2}(\Omega)}^{2}+\|p-\widehat{p}\|_{L^{2}\left(\Omega_{T}\right)}^{2}\right)
\end{aligned}
$$

with $C=C\left(\left\|z_{0}\right\|_{L^{\infty}(\Omega)},\left\|\widehat{z}_{0}\right\|_{L^{\infty}(\Omega)},\|p\|_{L^{\infty}\left(\Omega_{T}\right)} \wedge\|\widehat{p}\|_{L^{\infty}\left(\Omega_{T}\right)}\right)$. In particular, if we take $\widehat{z}_{0}=0, \widehat{p}=0$ in (3.15) and (3.17), which will imply $\widehat{z}^{\varepsilon}=0$, we have

$$
\begin{gathered}
\left\|z_{t}^{\varepsilon}\right\|_{L^{2}\left(0, T ; H_{\sigma}(\Omega)^{*}\right)}^{2}+\left\|z^{\varepsilon}\right\|_{C\left([0, T] ; L^{2}(\Omega)\right)}^{2}+\varepsilon\left\|\nabla z^{\varepsilon}\right\|_{L^{2}\left(\Omega_{T}\right)}^{2}+\left\|\sigma \nabla z^{\varepsilon}\right\|_{L^{2}\left(\Omega_{T}\right)}^{2} \\
\leq C\left\{\left\|z_{0}\right\|_{L^{2}(\Omega)}^{2}+\|p\|_{L^{1}\left(\Omega_{T}\right)}\right\}
\end{gathered}
$$


with $C=C\left(\left\|z_{0}\right\|_{L^{\infty}(\Omega)}\right)$. From (3.19),

$$
\begin{aligned}
& \left\|z_{t}^{\varepsilon}\right\|_{L^{2}\left(\Omega_{T}\right)}^{2}+\varepsilon\left\|\nabla z^{\varepsilon}\right\|_{C\left([0, T] ; L^{2}(\Omega)\right)}^{2}+\left\|\sigma \nabla z^{\varepsilon}\right\|_{C\left([0, T] ; L^{2}(\Omega)\right)}^{2} \\
& \leq \varepsilon\left\|\nabla z_{0}\right\|_{L^{2}(\Omega)}^{2}+C\left(\left\|\sigma \nabla z_{0}\right\|_{L^{2}(\Omega)}^{2}+\left\|z_{0}\right\|_{L^{2}(\Omega)}^{2}+\|p\|_{L^{2}\left(\Omega_{T}\right)}^{2}\right),
\end{aligned}
$$

with $C=C\left(\left\|z_{0}\right\|_{L^{\infty}(\Omega)}\right)$.

Now, we let $\left(z_{0}, p\right)$ be a pair of smooth data as above and let $z^{\varepsilon}$ be the corresponding solution of (3.7). By [3], we may assume that

$$
\lim _{\varepsilon \rightarrow 0}\left\|z^{\varepsilon}-z\right\|_{C\left(\bar{\Omega}_{T}\right)}=0
$$

where $z \in C\left(\bar{\Omega}_{T}\right)$ being the unique viscosity solution of (2.1). On the other hand, since $z^{\varepsilon}$ satisfies (3.18) and (3.19), and for any $\eta \in W_{2}^{0,1}\left(\Omega_{T}\right) \bigcap L^{2}\left(0, T ; H_{\sigma}(\Omega)\right)$ with $\left.\eta\right|_{t=T}=0$ and $\left.\eta\right|_{\partial \Omega}=0$, we have

$$
\begin{aligned}
\int_{\Omega_{T}}\left[-z^{\varepsilon} \eta_{t}\right. & \left.+\varepsilon \nabla z^{\varepsilon} \cdot \nabla \eta+\sum_{i, j=1}^{n} a_{i j} z_{x_{i}}^{\varepsilon} \eta_{x_{j}}\right] d x d t \\
= & \int_{\Omega} z_{0}^{\varepsilon}(x) \eta(x, 0) d x+\int_{\Omega_{T}}\left(a-b z^{\varepsilon}-p\right) z^{\varepsilon} \eta d x d t
\end{aligned}
$$

Together with (3.20) and (3.22), we may assume that $z \in L^{2}\left(0, T ; H_{\sigma}(\Omega)\right)$ with $z_{t} \in$ $L^{2}\left(0, T ; H_{\sigma}(\Omega)^{*}\right)$ and

$$
\begin{array}{ll}
z^{\varepsilon} \stackrel{w}{\longrightarrow} z, & \text { in } L^{2}\left(\Omega_{T}\right) \text { and in } L^{2}\left(0, T ; H_{\sigma}(\Omega)\right), \\
z_{t}^{\varepsilon} \stackrel{w}{\longrightarrow} z_{t}, & \text { in } L^{2}\left(0, T ; H_{\sigma}(\Omega)^{*}\right) .
\end{array}
$$

Pass to the limit in (3.23), we see that $z$ is also a solution of (2.1) in the sense of Definition 3.1. Then, taking limits in (3.12), (3.20) and (3.21), we get (3.2), (3.3) and (3.5) for this $z$.

Now, we let $\left(\widehat{z}_{0}, \widehat{p}\right)$ be another pair of smooth data and $\widehat{z}$ is the corresponding viscosity solution of (2.1), which is also a solution of (2.1) in the sense of Definition 3.1. Then, due to (3.15), (3.19) and the smoothness of the initial data and the controls, we see that (3.4) and (3.6) hold for $z$ and $\widehat{z}$.

Next, by density argument, we can show that for any $\left(z_{0}, p\right) \in \mathcal{Z} \times \mathcal{P}$, there exists a solution $z \in C\left([0, T] ; L^{2}(\Omega)\right) \bigcap L^{2}\left(0, T ; H_{\sigma}(\Omega)\right) \bigcap L^{\infty}\left(\Omega_{T}\right)$ with $z_{t} \in L^{2}\left(0, T ; H_{\sigma}(\Omega)^{*}\right)$ 
satisfying estimates (3.2)-(3.3) and the solutions $z$ and $\widehat{z}$ of $(2.1)$ corresponding to $\left(z_{0}, p\right)$, $\left(\widehat{z}_{0}, \widehat{p}\right) \in \mathcal{Z} \times \mathcal{P}$ obtained by the above limiting process satisfy the estimate (3.4). This proves (i), except the uniqueness, and (ii). Also, we can prove (iii) and (iv) similarly.

We should note that estimate (3.4) does not implies the uniqueness of the solutions because it is only satisfied by the solutions obtained by the above vanishing viscosity method. To prove the uniqueness, we let $z$ and $\widehat{z}$ be two solutions corresponding the pair $\left(z_{0}, p\right) \in \mathcal{Z}_{0} \times \mathcal{P}$. Set $w=\widehat{z}-z$. For any $t_{1} \in[0, T]$, we define

$$
\eta(x, t)=\left\{\begin{array}{l}
-\int_{t_{1}}^{t} w(x, s) d s, \quad \text { for } t \in\left[0, t_{1}\right), \\
0, \quad \text { for } t \in\left[t_{1}, T\right]
\end{array}\right.
$$

Clearly, $\eta \in L^{2}\left(0, T ; H_{\sigma}(\Omega)\right)$ and $\left.\eta\right|_{t=T}=0$. Also, $w=-\eta_{t}$. Now, by the definition of solutions, we have

$$
\int_{\Omega_{t_{1}}}\left\{\left(\eta_{t}\right)^{2}+\sigma \nabla w \cdot \sigma \nabla \eta\right\} d x d t=\int_{\Omega_{t_{1}}}[a-b(\widehat{z}+z)-p] w \eta d x d t .
$$

Then, it follows that

$$
\left\|\eta_{t}\right\|_{L^{2}\left(\Omega_{t_{1}}\right)}^{2}+\frac{1}{2}\|\sigma \nabla \eta(\cdot, 0)\|_{L^{2}(\Omega)} \leq C\left\|\eta_{t}\right\|_{L^{2}\left(\Omega_{t_{1}}\right)}\|\eta\|_{L^{2}\left(\Omega_{t_{1}}\right)}
$$

This gives

$$
\left\|\eta_{t}\right\|_{L^{2}\left(\Omega_{t_{1}}\right)}^{2} \leq C\|\eta\|_{L^{2}\left(\Omega_{t_{1}}\right)}^{2}
$$

Now, we let $\zeta(x, t)=\int_{0}^{t} w(x, \tau) d \tau$. Then, we see that for $t \in\left[0, t_{1}\right]$,

$$
\zeta_{t}=w=-\eta_{t}, \quad \eta(x, t)=\zeta\left(x, t_{1}\right)-\zeta(x, t)
$$

On the other hand,

$$
\left\|\zeta\left(\cdot, t_{1}\right)\right\|_{L^{2}(\Omega)}^{2}=2 \int_{\Omega} \int_{0}^{t_{1}} \zeta \zeta_{t} d t d x \leq\|\zeta\|_{L^{2}\left(\Omega_{t_{1}}\right)}^{2}+\left\|\zeta_{t}\right\|_{L^{2}\left(\Omega_{t_{1}}\right)}^{2} .
$$

Hence, combining (3.28)-(3.30), we obtain

$$
\begin{aligned}
& \left\|\zeta_{t}\right\|_{L^{2}\left(\Omega_{t_{1}}\right)}^{2} \leq C\|\eta\|_{L^{2}\left(\Omega_{t_{1}}\right)}^{2} \leq C\left\|\zeta\left(\cdot, t_{1}\right)\right\|_{L^{2}\left(\Omega_{t_{1}}\right)}^{2}+C\|\zeta\|_{L^{2}\left(\Omega_{t_{1}}\right)}^{2} \\
& \leq C t_{1}\left\|\zeta\left(\cdot, t_{1}\right)\right\|_{L^{2}(\Omega)}^{2}+C\|\zeta\|_{L^{2}\left(\Omega_{t_{1}}\right)}^{2} \leq C_{0} t_{1}\left\|\zeta_{t}\right\|_{L^{2}\left(\Omega_{t_{1}}\right)}^{2}+C\|\zeta\|_{L^{2}\left(\Omega_{t_{1}}\right)}^{2} \cdot
\end{aligned}
$$


The constant $C_{0}$ in the above only depends on the bounds of $z$ and $\widehat{z}$ and is independent of $t_{1} \in[0, T]$. We now choose $t_{1} \in(0, T]$ so that $C_{0} t_{1} \leq 1 / 2$. Then, we see that

$$
\left\|\zeta_{t}\right\|_{L^{2}\left(\Omega_{t}\right)}^{2} \leq C\|\zeta\|_{L^{2}\left(\Omega_{t}\right)}^{2}, \quad \forall t \in\left[0, t_{1}\right]
$$

¿From this, we have

$$
\int_{\Omega}|\zeta(x, t)|^{2} d x=2 \int_{0}^{t} \int_{\Omega} \zeta(x, s) \zeta_{t}(x, s) d x d s \leq C \int_{0}^{t} \int_{\Omega}|\zeta|^{2} d x d t, \quad t \in\left[0, t_{1}\right]
$$

Then, by Gronwall's inequality, we end up with $\zeta=0$ for $t \in\left[0, t_{1}\right]$. This implies $z=\widehat{z}$ for $t \in\left[0, t_{1}\right]$. Continuing this argument on $\left[t_{1}, 2 t_{1}\right]$ and so on, we can conclude that $z=\widehat{z}$ on $\Omega_{T}$.

Remark 3.3. We should note that in proving the above result, a key step is to obtain the strong convergence of $z^{\varepsilon}$ to $z$. This has been achieved via the notion of viscosity solutions. Here, we notice that condition (2.2) has not been used. From the result of $\S 4$, we will see that if condition (2.2) holds, which will imply the compactness of certain embedding, then we do not need the notion of viscosity solutions. See the proof of Theorem 5.2 .

Remark 3.4. Conclusion (3.2) means that whenever the initial state $z_{0}$ is strictly positive in $\Omega$, the state $z(x, t)$ will stay strictly positive in $\Omega$ for $t>0$. This fact is classical for the case of nondegenerate parabolic equations and for ordinary differential equations (corresponding to the case with $\sigma(x) \equiv 0$ ). Thus it is natural to have positivity of $z(x, t)>0(x, t) \in \Omega_{T}$ in our general case. We should point out that Theorem 3.2 also holds if $z_{0}$ is just nonnegative. To achieve this, we only need to make approximation of $z_{0}$ from above.

Remark 3.5. If the control $p \in C\left(\bar{\Omega}_{T}\right)$ and $z_{0} \in C(\bar{\Omega})$, then the state $z$ is in $C\left(\bar{\Omega}_{T}\right)$, by viscosity solution theory $([3])$.

\section{$\S 4$. Optimal Controls.}

In this section, we first present an existence of optimal control for our Problem $P_{K, M}$. The key result is the following 
Lemma 4.1. Let (A1)-(A3) holds. Then, for any $q>2$, the following imbedding is compact:

$$
L^{q}(\Omega) \bigcap H_{\sigma}(\Omega) \hookrightarrow L^{2}\left(\Omega_{T}\right)
$$

Proof. Let $\left\{y^{k}, k \geq 1\right\}$ be a sequence in $\left.L^{q}(\Omega) \bigcap H_{\sigma}(\Omega)\right)$ with

$$
\left\|y^{k}\right\|_{L^{q}(\Omega)},\left\|\sigma \nabla y^{k}\right\|_{L^{2}(\Omega)} \leq C, \quad \forall k \geq 1
$$

Without loss of generality, we assume that

$$
y^{k} \stackrel{w}{\longrightarrow} 0, \quad \text { in } L^{2}(\Omega), \quad(k \rightarrow \infty)
$$

We need to show that there exists a subsequence $\left\{y^{k_{m}}\right\}$ which strongly converges to 0 in $L^{2}(\Omega)$. To show this, we pick an $m \geq 1$. By $(2.2)$, there exists a $\delta_{m}>0$, such that

$$
\operatorname{meas}\left\{x \in \Omega \mid\left\{a_{i j}(x)\right\} \leq \delta_{m} I\right\}<\frac{1}{m} \text {. }
$$

Let us set

$$
\Omega^{m} \equiv\left\{x \in \Omega \mid\left\{a_{i j}(x)\right\}>\delta_{m} I\right\}
$$

which is an open set. By (4.2), we see that

$$
\left\|\nabla y^{k}\right\|_{L^{2}\left(\Omega^{m}\right)} \leq C, \quad \forall k \geq 1
$$

Hence, we may also assume that

$$
y^{k} \stackrel{s}{\rightarrow} 0, \quad \text { in } L^{2}\left(\Omega^{m}\right), \quad(k \rightarrow \infty) .
$$

Then, we have some $k_{m}$, with $k_{m}>k_{m-1}$, such that

$$
\left\|y^{k_{m}}\right\|_{L^{2}\left(\Omega^{m}\right)}<\frac{1}{m}
$$

Hence, combining (4.4) and (4.8), we end up with

$$
\begin{aligned}
\left\|y^{k_{m}}\right\|_{L^{2}(\Omega)} & \leq\left\|y^{k_{m}}\right\|_{L^{2}\left(\Omega^{m}\right)}+\left\|y^{k_{m}}\right\|_{L^{2}\left(\Omega \backslash \Omega^{m}\right)} \\
& \leq \frac{1}{m}+\left\|y^{k_{m}}\right\|_{L^{q}(\Omega)}\left(\frac{1}{m}\right)^{\frac{q-2}{2 q}}<\frac{1}{m}+C\left(\frac{1}{m}\right)^{\frac{q-2}{2 q}} .
\end{aligned}
$$


This completes the proof.

Our first main result of this section is the following theorem.

Theorem 4.2. Let assumptions (A1)-(A3) hold. Then, for any $K, M>0$, Problem $P_{K, M}$ admits at least one optimal control.

Proof. Let $\left\{p^{k}, k \geq 1\right\} \subset \mathcal{P}_{M}$ be a minimizing sequence and let $z^{k}$ be the state corresponding to $p^{k}$. Then, $p^{k}$ is bounded. Thus, by (3.3), we have that

$$
\left\|z_{t}^{k}\right\|_{L^{2}\left(0, T ; H_{\sigma}(\Omega)^{*}\right)}+\left\|z^{k}\right\|_{L^{2}\left(0, T ; H_{\sigma}(\Omega)\right)} \leq C . \quad \forall k \geq 1
$$

Then, we may assume that

$$
\begin{array}{lr}
z^{k} \stackrel{w}{\longrightarrow} \bar{z}, & \sigma \nabla z^{k} \stackrel{w}{\longrightarrow} \sigma \nabla \bar{z}, \quad \text { in } L^{2}\left(\Omega_{T}\right), \\
z_{t}^{k} \stackrel{w}{\longrightarrow} \bar{z}_{t}, & \text { in } L^{2}\left(0, T ; H_{\sigma}(\Omega)^{*}\right),
\end{array}
$$

for some $\bar{z} \in L^{2}\left(0, T ; H_{\sigma}(\Omega)\right)$ with $\bar{z}_{t} \in L^{2}\left(0, T ; H_{\sigma}(\Omega)^{*}\right)$ and also,

$$
p^{k} \stackrel{w}{\longrightarrow} \bar{p}, \quad \text { in } L^{2}\left(\Omega_{T}\right)
$$

for some $\bar{p} \in L^{2}\left(\Omega_{T}\right)$. On the other hand, by above Lemma 4.1 and [12, p.60, Proposition 4.2 , we may also assume that

$$
\lim _{k \rightarrow \infty}\left\|z^{k}-\bar{z}\right\|_{L^{2}\left(\Omega_{T}\right)}=0
$$

Then we see that $\bar{z}$ is the solution of (2.1) corresponding to $\bar{p}$. Moreover, by Fatou's lemma, we have

$$
J(\bar{p}) \leq \varliminf_{k \rightarrow \infty} J\left(p^{k}\right)=\inf _{p \in \mathcal{P}} J(p)
$$

Hence, $\bar{p}$ is an optimal control.

Now, let us derive the following optimality conditions for solutions to Problem $P_{K, M}$, by differentiating the cost functional $J(p)$ and the state function $z=z(p)$ with respect to the control $p$ at an optimal control. 
Theorem 4.3. Let (A1)-(A3) hold. Let $K, M>0$ and $z_{0} \in \mathcal{Z}$. Let $\bar{p} \in \mathcal{P}_{M}$ be an optimal control and $\bar{z}$ be the corresponding state for Problem $P_{K, M}$. Then, there exists a function $\lambda \in C\left([0, T] ; L^{2}(\Omega)\right) \cap L^{2}\left(0, T ; H_{\sigma}(\Omega)\right)$ satisfying the adjoint equation

$$
\left\{\begin{array}{l}
-\lambda_{t}-\sum_{i, j=1}^{n}\left(a_{i j} \lambda_{x_{i}}\right)_{x_{j}}=(a-2 b \bar{z}-r-\bar{p}) \lambda+\alpha \bar{z}+\beta \bar{p}^{2}, \quad \text { in } \Omega_{T}, \\
\left.\lambda\right|_{\partial \Omega}=0 \\
\left.\lambda\right|_{t=T}=0
\end{array}\right.
$$

such that the optimal control $\bar{p}$ satisfies

$$
\bar{p}=\min \left\{M, \frac{\lambda}{2 \beta}\right\}, \quad \text { on } \Omega_{T} .
$$

Moreover, if

$$
M \geq \frac{\alpha}{2 \beta} e^{(a-r) T}\left(\frac{a}{b} \vee K\right),
$$

then,

$$
\bar{p}=\frac{\lambda}{2 \beta}, \quad \text { on } \Omega_{T} .
$$

Proof. Let $\bar{p} \in \mathcal{P}_{M}$ be an optimal control and $\bar{z}$ be the corresponding state. Let $h \in L^{\infty}$ such that

$$
\bar{p}+\varepsilon h \in \mathcal{P}_{M}, \quad \text { for all sufficiently small } \varepsilon>0 .
$$

Let $z^{\varepsilon}$ be the state corresponding to $\bar{p}+\varepsilon h$. Then, by (3.4), we have $z^{\varepsilon} \stackrel{s}{\rightarrow} \bar{z}$ in $L^{2}(0, T$; $\left.H_{\sigma}(\Omega)\right)$ and the function $\xi^{\varepsilon}=\frac{1}{\varepsilon}\left(z^{\varepsilon}-\bar{z}\right)$ satisfies the following:

$$
\left\{\begin{array}{l}
\xi_{t}^{\varepsilon}-\sum_{i, j=1}^{n}\left(a_{i j} \xi_{x_{i}}^{\varepsilon}\right)_{x_{j}}=\xi^{\varepsilon}\left[a-b\left(z^{\varepsilon}+\bar{z}\right)-\bar{p}\right]-h z^{\varepsilon}, \quad \text { in } \Omega_{T}, \\
\left.\xi^{\varepsilon}\right|_{\partial \Omega}=0, \\
\left.\xi^{\varepsilon}\right|_{t=0}=0 .
\end{array}\right.
$$

By the results of $\S 3$, we see that

$$
\left\|\xi_{t}^{\varepsilon}\right\|_{L^{2}\left(0, T ; H_{\sigma}(\Omega)^{*}\right)}+\left\|\xi^{\varepsilon}\right\|_{L^{2}\left(\Omega_{T}\right)}+\left\|\sigma \nabla \xi^{\varepsilon}\right\|_{L^{2}\left(\Omega_{T}\right)} \leq C, \quad \forall \varepsilon>0 .
$$


Hence, we may assume that for some $\xi \in L^{2}\left(0, T ; H_{\sigma}(\Omega)\right)$ with $\xi_{t} \in L^{2}\left(0, T ; H_{\sigma}(\Omega)^{*}\right)$,

$$
\begin{array}{ll}
\xi^{\varepsilon} \stackrel{w}{\longrightarrow} \xi, & \text { in } L^{2}\left(0, T ; H_{\sigma}(\Omega)\right), \\
\xi_{t}^{\varepsilon} \stackrel{w}{\longrightarrow} \xi_{t}, & \text { in } L^{2}\left(0, T ; H_{\sigma}(\Omega)^{*}\right) .
\end{array}
$$

Since the equation for $\xi^{\varepsilon}$ is linear, passing to the limit in (4.20) shows that $\xi$ is the unique solution of the variational system:

$$
\left\{\begin{array}{l}
\xi_{t}-\sum_{i, j=1}^{n}\left(a_{i j} \xi_{x_{i}}\right)_{x_{j}}=\xi[a-2 b \bar{z}-\bar{p}]-h \bar{z}, \quad \text { in } \Omega_{T} \\
\left.\xi\right|_{\partial \Omega}=0 \\
\left.\xi\right|_{t=0}=0 .
\end{array}\right.
$$

Next, by the optimality of $\bar{p}$, we have

$$
\begin{aligned}
0 & \leq \lim _{\varepsilon \rightarrow 0} \frac{J(\bar{p}+\varepsilon h)-J(\bar{p})}{\varepsilon} \\
& =\int_{\Omega_{T}} e^{-r t}\left\{\left(\alpha \bar{z}+\beta \bar{p}^{2}\right) \xi+2 \beta \bar{z} \bar{p} h\right\} d x d t .
\end{aligned}
$$

We let $\lambda$ be the unique solution of (4.15). The existence and uniqueness of such a solution follows from a similar argument of $\S 3$. Then, substituting from the adjoint equation into (4.24) and integrating by parts gives (see [9])

$$
\int_{\Omega_{T}} e^{-r t} \bar{z} h(2 \beta \bar{p}-\lambda) d x d t \geq 0 .
$$

Now, let us make some observations on the above. First of all, since

$$
\alpha \bar{z}+\beta \bar{p}^{2} \geq 0,
$$

by maximum principle, we see that

$$
\bar{\lambda} \geq 0 .
$$

Next, by definition of $\mathcal{Z}$ (see $\S 2$ ), since $z_{0} \in \mathcal{Z}$, it is strictly positive in $\Omega$. By (3.2), the optimal state $\bar{z}$ is strictly positive in $\Omega_{T}$. Now, on the set $\{\bar{p}=0\}$, we may choose nonnegative $h$ with support contained in this set. Then, (4.25) and (4.27) lead

$$
\lambda=0, \quad \text { on }\{\bar{p}=0\} .
$$


On the set $\{\bar{p}=M\}$, we may choose non-positive $h$ with support contained in this set. Then, (4.25) gives

$$
M=\bar{p} \leq \frac{\lambda}{2 \beta}, \quad \text { on }\{\bar{p}=M\}
$$

Using $h$ with arbitrary sign, we clearly have that

$$
\bar{p}=\frac{\lambda}{2 \beta}, \quad \text { on }\{0<\bar{p}<M\}
$$

Thus, combining the above, we obtain (4.16). Next, we let $M$ and $K$ satisfy (4.17). By $(4.15)-(4.16)$, we see that

$$
\beta \bar{p}^{2}-\bar{p} \lambda=\beta \bar{p}\left(\bar{p}-\frac{\lambda}{\beta}\right) \leq 0 .
$$

We let $\Lambda$ be the solution of the following problem:

$$
\left\{\begin{array}{l}
-\Lambda_{t}-\sum_{i, j=1}^{n}\left(a_{i j} \Lambda_{x_{i}}\right)_{x_{j}}=(a-r) \Lambda+\alpha \bar{z}, \quad \text { in } \Omega_{T} \\
\left.\Lambda\right|_{\partial \Omega}=0 \\
\left.\Lambda\right|_{t=T}=0
\end{array}\right.
$$

By maximum principle, we have that

$$
0 \leq \lambda \leq \Lambda, \quad \text { in } \Omega_{T}
$$

On the other hand, we can prove that (note (3.2))

$$
\begin{gathered}
0 \leq \Lambda(x, t) \leq e^{(a-r) T} \alpha\|\bar{z}\|_{L^{\infty}\left(\Omega_{T}\right)} \leq \alpha e^{(a-r) T}\left(\frac{a}{b} \vee\left\|z_{0}\right\|_{L^{\infty}(\Omega)}\right) \leq 2 \beta M \\
(x, t) \in \Omega_{T}
\end{gathered}
$$

Then, it follows that

$$
\frac{\lambda}{2 \beta} \leq M
$$

Hence, (4.18) follows.

Remark 4.4. We see that in proving Theorem 4.4, the condition (2.2) is not needed. 
Now, we let $z$ is an optimal state for the Problem $P_{K, M}$ with $K$ and $M$ satisfying (4.17). Let $\lambda$ be the solution of (4.15) with $\bar{p} \in \mathcal{P}_{M}$ being an optimal control satisfying (4.18). Then, substituting the characterization of the optimal control, (4.18), into (2.1) and (4.15), we obtain the following coupled two-point boundary value problem:

$$
\left\{\begin{array}{l}
z_{t}-\sum_{i, j=1}^{n}\left(a_{i j} z_{x_{i}}\right)_{x_{j}}=a z-b z^{2}-\frac{\lambda}{2 \beta} z \\
-\lambda_{t}-\sum_{i, j=1}^{n}\left(a_{i j} \lambda_{x_{i}}\right)_{x_{j}}=a \lambda-2 b z \lambda-r \lambda-\frac{\lambda^{2}}{4 \beta}+\alpha z \\
\left.z\right|_{\partial \Omega}=\left.\lambda\right|_{\partial \Omega}=0 \\
\left.z\right|_{t=0}=z_{0} \\
\left.\lambda\right|_{t=T}=0 .
\end{array}\right.
$$

This system is called the optimality system for our Problem $P_{K, M}$. By the "existence of an optimal control" result, we know that (4.36) admits a solution $(z, \lambda)$. In the rest of this section, we prove that for $T$ is small, the solution to the above optimality system is unique. In [9], such a result was proved for nondegenerate case. Let us now state and prove our result.

Theorem 4.5. Let (A1)-(A3) hold. Then, provided $T>0$ is small enough, (4.36) admits at most one solution.

Proof. Suppose $(z, \lambda)$ and $(\widehat{z}, \widehat{\lambda})$ are two solutions. Set

$$
\begin{aligned}
& w=\widehat{z}-z, \quad v=\widehat{\lambda}-\lambda, \\
& \eta(x, t)=\int_{t}^{T} w(x, s) d s, \quad q(x, t)=\int_{0}^{t} v(x, s) d s .
\end{aligned}
$$

Then, we see that

$$
\eta(x, T)=0, \quad q(x, 0)=0 ; \quad \eta_{t}=-w, \quad q_{t}=w .
$$

Using $\eta$ and $q$ as test functions in the equations for $w$ and $v$, respectively, we obtain

$$
\begin{aligned}
& \int_{\Omega_{T}}\left[w\left(-\eta_{t}\right)+v q_{t}+a_{i j} w_{x_{i}} \eta_{x_{j}}+a_{i j} v_{x_{i}} q_{x_{j}}\right] \\
& =\int_{\Omega_{T}}\left[a w \eta-b(\widehat{z}+z) w \eta-\frac{1}{2 \beta}(\lambda w+v \widehat{z}) \eta+(a-r) v q\right. \\
& \left.\quad-2 b(w \lambda+v \widehat{z}) q-\frac{1}{4 \beta}(\widehat{\lambda}+\lambda) v q+\alpha w q\right] .
\end{aligned}
$$


Then,

$$
\begin{aligned}
& \left\|\eta_{t}\right\|_{L^{2}\left(\Omega_{T}\right)}^{2}+\left\|q_{t}\right\|_{L^{2}\left(\Omega_{T}\right)}^{2}+\frac{1}{2}\|\sigma \nabla \eta(\cdot, 0)\|_{L^{2}(\Omega)}^{2}+\frac{1}{2}\|\sigma \nabla q(\cdot, T)\|_{L^{2}(\Omega)}^{2} \\
& \leq C\left\{\left\|\eta_{t}\right\|_{L^{2}\left(\Omega_{T}\right)}\|\eta\|_{L^{2}\left(\Omega_{T}\right)}+\left\|q_{t}\right\|_{L^{2}\left(\Omega_{T}\right)}\|\eta\|_{L^{2}\left(\Omega_{T}\right)}\right. \\
& \left.\quad+\left\|\eta_{t}\right\|_{L^{2}\left(\Omega_{T}\right)}\|q\|_{L^{2}\left(\Omega_{T}\right)}+\left\|q_{t}\right\|_{L^{2}\left(\Omega_{T}\right)}\|q\|_{L^{2}\left(\Omega_{T}\right)}\right\} .
\end{aligned}
$$

This yields

$$
\left\|\eta_{t}\right\|_{L^{2}\left(\Omega_{T}\right)}^{2}+\left\|q_{t}\right\|_{L^{2}\left(\Omega_{T}\right)}^{2} \leq C\left\{\|\eta\|_{L^{2}\left(\Omega_{T}\right)}^{2}+\|q\|_{L^{2}\left(\Omega_{T}\right)}^{2}\right\}
$$

Next, we let

$$
\zeta(x, t)=\int_{0}^{t} w(x, s) d s
$$

Then,

$$
\zeta_{t}=w=-\eta_{t}, \quad \eta(x, t)=\zeta(x, T)-\zeta(x, t)
$$

and

$$
\|\zeta(\cdot, T)\|_{L^{2}(\Omega)}^{2}=2 \int_{\Omega} \int_{0}^{T} \zeta \zeta_{t} d x d t \leq\|\zeta\|_{L^{2}\left(\Omega_{T}\right)}^{2}+\left\|\zeta_{t}\right\|_{L^{2}\left(\Omega_{T}\right)}^{2} .
$$

Then, by (4.41) and (4.43), we have

$$
\begin{aligned}
& \left\|\zeta_{t}\right\|_{L^{2}\left(\Omega_{T}\right)}^{2}+\left\|q_{t}\right\|_{L^{2}\left(\Omega_{T}\right)}^{2} \leq C\left\{\|\eta\|_{L^{2}\left(\Omega_{T}\right)}^{2}+\|q\|_{L^{2}\left(\Omega_{T}\right)}^{2}\right\} \\
& \leq C\|\zeta(\cdot, T)\|_{L^{2}\left(\Omega_{T}\right)}^{2}+C\|\zeta\|_{L^{2}\left(\Omega_{T}\right)}^{2}+C\|q\|_{L^{2}\left(\Omega_{T}\right)}^{2} \\
& \leq C T\|\zeta(\cdot, T)\|_{L^{(\Omega)}}^{2}+C\left(\|\zeta\|_{L^{2}\left(\Omega_{T}\right)}^{2}+\|q\|_{L^{2}\left(\Omega_{T}\right)}^{2}\right) \\
& \leq C_{0} T\left\|\zeta_{t}\right\|_{L^{2}\left(\Omega_{T}\right)}^{2}+C\left(\|\zeta\|_{L^{2}\left(\Omega_{T}\right)}^{2}+\|q\|_{L^{2}\left(\Omega_{T}\right)}^{2}\right)
\end{aligned}
$$

with $C_{0}=C_{0}(T)$ being nondecreasing in $T$. Then, for $T$ sufficiently small, we obtain

$$
\left\|\zeta_{t}\right\|_{L^{2}\left(\Omega_{T}\right)}^{2}+\left\|q_{t}\right\|_{L^{2}\left(\Omega_{T}\right)}^{2} \leq C\left\{\|\zeta\|_{L^{2}\left(\Omega_{T}\right)}^{2}+\|q\|_{L^{2}\left(\Omega_{T}\right)}^{2}\right\}
$$

Next, we consider the following:

$$
\begin{aligned}
& \|\zeta\|_{L^{2}\left(\Omega_{T}\right)}^{2}=\int_{\Omega} \int_{0}^{T}\left(\int_{0}^{t} \zeta_{t}(x, s) d s\right)^{2} d x d t \\
& \leq \int_{\Omega} \int_{0}^{T} t \int_{0}^{t} \zeta_{t}(x, s)^{2} d s d x d t \leq \frac{T^{2}}{2}\left\|\zeta_{t}\right\|_{L^{2}\left(\Omega_{T}\right)}^{2}
\end{aligned}
$$


We have the same thing for $q$. Thus, (4.46) implies

$$
\left\|\zeta_{t}\right\|_{L^{2}\left(\Omega_{T}\right)}^{2}+\left\|q_{t}\right\|_{L^{2}\left(\Omega_{T}\right)}^{2} \leq C T^{2}\left\{\left\|\zeta_{t}\right\|_{L^{2}\left(\Omega_{T}\right)}^{2}+\left\|q_{t}\right\|_{L^{2}\left(\Omega_{T}\right)}^{2}\right\}
$$

with $C=C(T)$ being nondecreasing in $T$. Hence, for small $T$, the above yields

$$
\|\zeta\|_{L^{2}\left(\Omega_{T}\right)}^{2}+\|q\|_{L^{2}\left(\Omega_{T}\right)}^{2}=0
$$

This gives $\widehat{z}=z$ and $\widehat{\lambda}=\lambda$.

Remark 4.6. Due to the opposite orientation of the state equation and the adjoint equation, we cannot stack the intervals as in the proof of Theorem 3.2 to get uniqueness of solutions for large $T$.

Remark 4.7. Combining the results of Theorems 4.2, 4.3 and 4.5, we see that the optimal control, the proportion of the nuisance population to be trapped, is uniquely characterized by the solution of the optimality system.

\section{$\S 5$. Problems for Neumann Boundary Conditions.}

In this section, we will study the problem with Neumann type boundary conditions. In principle, the results are almost the same. Thus, we will concentrate on the difference between these two problems.

For convenience, we write down the state equation as follows:

$$
\left\{\begin{array}{l}
z_{t}-\sum_{i, j=1}^{n}\left(a_{i j}(x) z_{x_{i}}\right)_{x_{j}}=z(a-b z-p), \quad \text { in } \Omega_{T} \\
\left.\frac{\partial z}{\partial \nu_{a}}\right|_{\partial \Omega}=0 \\
\left.z\right|_{t=0}=z_{0}(x)
\end{array}\right.
$$

where $\frac{\partial}{\partial \nu_{a}}$ is the conormal derivative on the boundary $\partial \Omega$ compatible with the matrix $\left\{a_{i j}(x)\right\}$. Namely,

$$
\frac{\partial z}{\partial \nu_{a}}=\left(\sigma^{*} \sigma \nabla z\right) \cdot \nu
$$

with $\nu$ being the outward normal to $\partial \Omega$. In studying the above state equation, similar to $\S 2$, we define the space $\bar{H}_{\sigma}(\Omega)$ to be the completion of $C^{\infty}(\bar{\Omega})$ under norm $\|\cdot\|_{H_{\sigma}(\Omega)}$ defined 
by (2.4). Also, we let $\bar{H}_{\sigma}(\Omega)^{*}$ be the dual of $\bar{H}_{\sigma}(\Omega)$. Next, we introduce the following definition of solutions to the state equation.

Definition 5.1. Let $\left(z_{0}, p\right) \in \mathcal{Z} \times \mathcal{P}$ be given. A function $z \in L^{2}\left(0, T ; \bar{H}_{\sigma}(\Omega)\right)$ is called a solution of (5.1) if the following holds:

$$
\begin{gathered}
\int_{\Omega_{T}}\left[-z \eta_{t}+\sum_{i, j=1}^{n} a_{i j} z_{x_{i}} \eta_{x_{j}}\right] d x d t=\int_{\Omega} z_{0}(x) \eta(x, 0) d x+\int_{\Omega_{T}}(a-b z-p) z \eta d x d t \\
\forall \eta \in W_{2}^{0,1}\left(\Omega_{T}\right) \bigcap L^{2}\left(0, T ; \bar{H}_{\sigma}(\Omega)\right), \quad \text { with }\left.\eta\right|_{t=T}=0 .
\end{gathered}
$$

Our result about the well-posedness of the state equation can be stated as follows.

Theorem 5.2. Let (A1)-(A2) hold. Then, the conclusion of Theorem 3.2 holds with $H_{\sigma}(\Omega)$ being replaced by $\bar{H}_{\sigma}(\Omega)$ and (2.1) being replaced by (5.1).

Sketch of the Proof. Here, we sketch a proof by using condition (2.2) and without using the notion of viscosity solutions. To this end, we introduce the following approximating problem:

$$
\left\{\begin{array}{l}
z_{t}^{\varepsilon}-\varepsilon \Delta z^{\varepsilon}-\sum_{i, j=1}^{n}\left(a_{i j}(x) z_{x_{i}}^{\varepsilon}\right)_{x_{j}}=z^{\varepsilon}\left(a-b z^{\varepsilon}-p\right), \quad \text { in } \Omega_{T}, \\
\left.\left\{\left[\left(\varepsilon I+\sigma^{*} \sigma\right) \nabla z^{\varepsilon}\right] \cdot \nu\right\}\right|_{\partial \Omega}=0, \\
\left.z^{\varepsilon}\right|_{t=0}=z_{0}(x) .
\end{array}\right.
$$

Here, we should note that the corresponding boundary condition has been taken as the natural one (compatible with the differential operator). This problem admits a unique classical solution $z^{\varepsilon}$. Similar to $\S 3$, we are able to obtain estimates $(3.8),(3.12),(3.15)$, (3.17) and (3.19)-(3.21), with $H_{\sigma}(\Omega)$ being replaced by $\bar{H}_{\sigma}(\Omega)$. Also, $(3.23)$ holds for any $\eta \in W_{2}^{0,1}\left(\Omega_{T}\right) \bigcap L^{2}\left(0, T ; H_{\sigma}(\Omega)\right)$ with $\left.\eta\right|_{t=T}=0$. Then, by (2.2), Lemma 4.1 and estimates (3.8) and (3.20), we can assume that

$$
\begin{aligned}
& z^{\varepsilon} \stackrel{s}{\rightarrow} z, \quad \text { in } L^{2}\left(\Omega_{T}\right), \\
& \sigma \nabla z^{\varepsilon} \stackrel{w}{\longrightarrow} \sigma \nabla z, \quad \text { in } L^{2}\left(\Omega_{T}\right) .
\end{aligned}
$$

Then, notice that (by $(3.20)$ )

$$
\varepsilon\left\|\nabla z^{\varepsilon}\right\|_{L^{2}\left(\Omega_{T}\right)} \rightarrow 0
$$


we can pass to the limits in (3.23) to get the existence of a solution. The estimates (3.2)(3.6) follow immediately. The proof of the uniqueness is also similar to Theorem 3.2. Thus, we have completed the sketch.

We have seen that the major difference is that in the approximating state equation (5.3), we should choose the boundary condition compatible with the second order operator to obtain the required estimates for the approximating solution $z^{\varepsilon}$ of the state equation.

Now, we have that for each $\left(z_{0}, p\right) \in \mathcal{Z} \times \mathcal{P}$, there exists a unique corresponding state $z$. Thus, we can define the cost functional as (2.6). Then, for each pair $(K, M) \in$ $(0, \infty) \times(0, \infty)$, we can pose the optimal control problem as in $\S 2$. Let us refer to this problem as Problem $\bar{P}_{K, M}$. Next, similar to Theorem 4.2, we are able to prove the existence of optimal control for Problem $\bar{P}_{K, M}$. We are not going to repeat it here. Finally, let us state the following optimality conditions for optimal controls.

Theorem 5.2. Let (A1)-(A3) hold. Let $K, M>0$ and $z_{\in} \mathcal{Z}$. Let $\bar{p} \in \mathcal{P}_{M}$ be an optimal control with $\bar{z}$ being the corresponding state for Problem $\bar{P}_{K, M}$. Then, there exists a function $\lambda \in C\left([0, T] ; L^{2}(\Omega)\right) \bigcap L^{2}\left(0, T ; \bar{H}_{\sigma}(\Omega)\right)$ satisfying the adjoint equation

$$
\left\{\begin{array}{l}
-\lambda_{t}-\sum_{i, j=1}^{n}\left(a_{i j} \lambda_{x_{i}}\right)_{x_{j}}=(a-2 b \bar{z}-r-\bar{p}) \lambda+\alpha \bar{z}+\beta \bar{p}^{2}, \quad \text { in } \Omega_{T}, \\
\left.\frac{\partial \lambda}{\partial \nu_{a}}\right|_{\partial \Omega}=0, \\
\left.\lambda\right|_{t=T}=0,
\end{array}\right.
$$

such that the optimal control $\bar{p}$ satisfies

$$
\bar{p}=\min \left\{M, \frac{\lambda}{2 \beta}\right\}, \quad \text { on } \Omega_{T}
$$

Moreover, if

$$
M \geq \frac{\alpha}{2 \beta} e^{(a-r) T}\left(\frac{a}{b} \vee K\right)
$$

then,

$$
\bar{p}=\frac{\lambda}{2 \beta}, \quad \text { on } \Omega_{T}
$$


The proof is the same as that of Theorem 4.3. We also have an analogous result as Theorem 4.5. We omit the details here.

Remark 5.3. In proving Theorem 5.2, condition (2.2) can be removed. Through the viscosity solution theory for Neumann problems $([3])$, one can obtain the strong convergence of $z^{\varepsilon}$ to $z$ without using the compactness result, Lemma 4.1, which requires (2.2).

\section{References}

[1] M. Chipot, Variational Inequalities and Flow in Porous Media, Springer-Verlag, New York, 1984.

[2] C. Clark, Mathematical Bioeconomics: The Optimal Management of Renewable Resources, second edition, Wiley Interscience, New York, 1990.

[3] M. G. Crandall, H. Ishii and P. L. Lions, User's guide to viscosity solutions of second order partial differential equations, Bull. Amer. Math. Soc. (New Series), 27 (1992), $1-67$.

[4] P. Fife, Mathematical Aspects of Reacting and Diffusing Systems, Lecture Notes in Biomathematics no.28, Springer-Verlag, New York, 1979.

[5] F. He, A. Leung, and S. Stojanovic, Periodic optimal control for competing parabolic Volterra-Lotka type systems, preprint.

[6] E. P. Hill, Beaver, Wild Mammals of North America, Biology, Management, and Economics, ed. J. A. Chapman and G. A. Feldhamer, John Hopkins Univ. Press, Baltimore, 1982 .

[7] O. A. Ladyzenskaja, V. A. Solonnikov and N. N. Ural'ceva, Linear and Quasilinear Equations of Parabolic Type, Amer. Math. Soc., Providence, R.I., 1968.

[8] R. A. Lancia and J. W. Bishir, Mortality rates of beaver in Newfoundland: A comment, J. Wildlife Management, 49 (1985), 879-881.

[9] S. M. Lenhart and M.G. Bhat, Application of distributed parameter control model in wildlife damage management, Math. Models \& Methods in Appl. Sci., to appear. 
[10] A. Leung and S. Stojanovic, Optimal control for elliptic Volterra-Lotka equations, J. Math. Anal. \& Appl. to appear.

[11] J. L. Lions, Equations Differentielles Operationnelles et Problèmes aux Limites, Springer-Verlag, Berlin, 1961.

[12] J. L. Lions, Optimal Control of Systems Governed by Partial Differential Equations, Springer-Verlag, New York, 1971.

[13] J. E. Miller, Assessment of wildlife damage on southern forests, Proceedings of the American Foresters' Annual Meeting, Alabama, 1986.

[14] J. D. Murray, Mathematical Biology, Springer-Verlag, New York, 1989.

[15] O. A. Oleinik and E. V. Radkevic, Second order Equations with Nonnegative characteristic Forms, Amer. Math. Soc., Plenum Press, New York, 1973.

[16] J. G. Skellam, Random dispersal in theoretical populations, Biometrica, 38 (1951), $196-218$. 